livraisons

d'Histoire

de l'Architecture

\section{Livraisons de l'histoire de l'architecture}

$30 \mid 2015$

Le dessin d'architecture : œuvre/outil des architectes

?

\title{
Aujourd'hui à Chlef le centre Larbi Tebessi, hier à Orléansville le centre Albert-Camus (1955-1961), (architectes Louis Miquel et Roland Simounet)
}

Today in Chlef the center "Larbi Tebessi", yesterday in Orléansville the center

"Albert-Camus" (1955-1961), (architects Louis Miquel and Roland Simounet)

Heute in Chlef das Centre Larbi Tebessi, gestern in Orléansville das Centre

Albert-Camus (1955-1961), Werke der Architekten Louis Miquel und Roland

\section{Soraya Bertaud du Chazaud}

\section{OpenEdition}

Journals

Édition électronique

URL : http://journals.openedition.org/lha/560

DOI : $10.4000 /$ /ha. 560

ISSN : 1960-5994

Éditeur

Association Livraisons d'histoire de l'architecture - LHA

Édition imprimée

Date de publication : 30 décembre 2015

Pagination : 117-129

ISSN : 1627-4970

\section{Référence électronique}

Soraya Bertaud du Chazaud, « Aujourd'hui à Chlef le centre Larbi Tebessi, hier à Orléansville le centre Albert-Camus (1955-1961), (architectes Louis Miquel et Roland Simounet) », Livraisons de l'histoire de l'architecture [En ligne], 30 | 2015, mis en ligne le 18 décembre 2017, consulté le 10 décembre 2020. URL : http://journals.openedition.org//ha/560 ; DOI : https://doi.org/10.4000//ha.560 
Par Soraya DU CHAZAUD

\section{AUJOURD'HUI À CHLEF LE CENTRE LARBI TEBESSI, HIER À ORLÉANSVILLE LE CENTRE ALBERT-CAMUS (1955-1961 - ARCHITECTES LOUIS MIQUEL ET ROLAND SIMOUNET)}

Après des recherches sur l'Aéro-habitat de Louis Miquel à Alger, sujet d'un premier mémoire de Master 1 à l'université Paris 1 Panthéon-Sorbonne, mon intérêt pour l'architecture moderne en Algérie s'est accru. C'est en consultant le numéro 329 de la revue Technique et architecture, qui consacre pour les mois de février-mars 1980 un numéro spécial sur l'Algérie, que je découvre le centre culturel Albert-Camus d'Orléansville. Bâti entre 1955 et 1961, il survit au tremblement de terre meurtrier et dévastateur de 1980 à El-Asnam, nom donné à la ville après l'indépendance. Aujourd'hui la ville s'appelle Chlef, et le centre "Larbi Tebessi ».

Depuis mon précédent travail sur l'Aéro-habitat, je connais les difficultés à ouvrir les archives algériennes. Nombre d'architectes ont quitté l'Algérie en y laissant leurs archives, c'est le cas de Louis Miquel et de Roland Simounet, et elles ont disparu. En France, les archives de ces deux architectes sont facilement accessibles, celles de Miquel à l'Institut français d'architecture à Paris et celles de Simounet aux Archives nationales du monde du travail à Roubaix. Après enquête, j’ai pu être assurée que celles-ci sont nourries de ce projet d'Orléansville.

Pour parler objectivement, sinon le plus complètement possible, d'un bâtiment, deux visites se sont imposées en 2012 et en 2014 pour effectuer cette " promenade architecturale " chère à Le Corbusier, et noter son évolution et sa réception actuelle. Mes problématiques sont restées les mêmes que celles posées pour l'Aéro-habitat, à savoir : la question de la conservation de ce patrimoine culturel des années 1950, la question de l'héritage d'une architecture réservée à la population française sur un territoire algérien et la question du transfert de ce lieu culturel et sportif après l'indépendance.

\section{Un contexte particulier}

Le séisme du 9 septembre 1954

Les 9 et 10 septembre 1954, un tremblement de terre provoque la destruction quasi totale de la ville. La ville est détruite à $80 \%$, le bilan est lourd : 1600 morts, 5000 blessés, 65000 maisons détruites dont 25000 très endommagées.

À la veille du séisme, Orléansville est une ville moyenne, réputée pour ses vergers, dont la cité et les faubourgs comptent 40000 habitants, parmi lesquels 
5000 européens occupant le centre-ville. Il est à l'image d'une ville provinciale du midi de la France conçue pour les européens.

Les Algériens se répartissent depuis 1900 deux quartiers extérieurs : la "Ferme » au nord et "Bokat Sahnoun" au sud. La résorption de ces quartiers surpeuplés sera une des priorités lors de la reconstruction de la ville. Il faut donc attendre le tremblement de terre de septembre 1954 pour qu'Orléansville " accepte » de s'agrandir et de reloger toutes les populations, quelles que soient leurs origines.

À la suite de cette catastrophe, un décret du 6 octobre 1954 met en place un Commissariat à la reconstruction dépendant du Gouvernement général et piloté par Jean de Maisonseul, alors directeur du service départemental d'urbanisme de la région d'Alger. Le projet de reconstruction est intéressant dans la mesure où il est un exemple d'élaboration d'un plan de ville nouvelle comme ce fut le cas pour la reconstruction des villes bombardées après la Seconde Guerre mondiale.

Venus d'Alger, des groupes d'architectes aux doctrines opposées sont en concurrence: Fernand Pouillon, l'agence du Plan d'Alger et une école "corbuséenne " algéroise composée d'élèves ou d'admirateurs du "maitre ». Pour des raisons politiques, Fernand Pouillon est écarté du projet de reconstruction dévolu aux architectes des deux autres tendances.

Le Gouvernement général veut mettre en œuvre une reconstruction rapide et exemplaire. Dès le lendemain du tremblement de terre, Jean de Maisonseul est à Orléansville pour mesurer la gravité des dommages. La ville est désormais sous l'autorité de son service, et Jean de Maisonseul détient les pleins pouvoirs sur le choix de l'équipe d'architectes qui devra se charger de cette tâche. De plus, le contexte politique étant difficile en ce début de guerre d'Algérie, on lui permet de travailler dans le secret le plus complet, sans subir aucune influence politique locale. Quarantecinq architectes d'opération sont recrutés : des architectes locaux venant de toutes les tendances algéroises arrivent les premiers, suivis d'autres venant de France.

Pour superviser la reconstruction, on fait dans un premier temps appel à André Ravéreau ${ }^{1}$. Il reste peu à Orléansville. Il est appelé sur l'île grecque de Céphalonie, également dévastée un an auparavant, en 1953, par un tremblement de terre. Pour lui succéder, Jean Bossu, ancien collaborateur de Le Corbusier, est nommé architecte en chef de la reconstruction d'Orléansville en juillet 1955. Il est choisi pour sa neutralité, son impartialité et surtout son regard neuf sur l'Algérie : il n'appartient ni au «camp » de l'agence du Plan, ni à celui de Pouillon, ni aux corbuséens algérois.

Il récupère le projet du centre de commerces et d'habitations Saint-Réparatus (ill. 1) qu'il construit entre 1955 et 1964 avec ce qu'il appelle "le produit rouge ", la brique s'adaptant au climat et se confondant au paysage environnant, ce qui en fait sa singularité. Contrairement aux autres architectes européens, Jean Bossu fait une architecture attentive au site, notamment après son voyage au M'zab. Le centre Saint-Réparatus succombera au second tremblement de terre de 1980.

1. L'architecte André Ravéreau est arrivé en Algérie en 1954 pour la reconstruction d'Orléansville et la quitte en 1976. Il fut architecte en chef des monuments historiques en Algérie de 1965 à 1971. En 1970 il créa l'Atelier d'étude et de restauration de la vallée du M'zab. Il construit dans le M'zab une architecture adaptée au site, au climat, aux usages.

Livraisons J'bistoire de l'architecture $n^{\circ} 30$ 


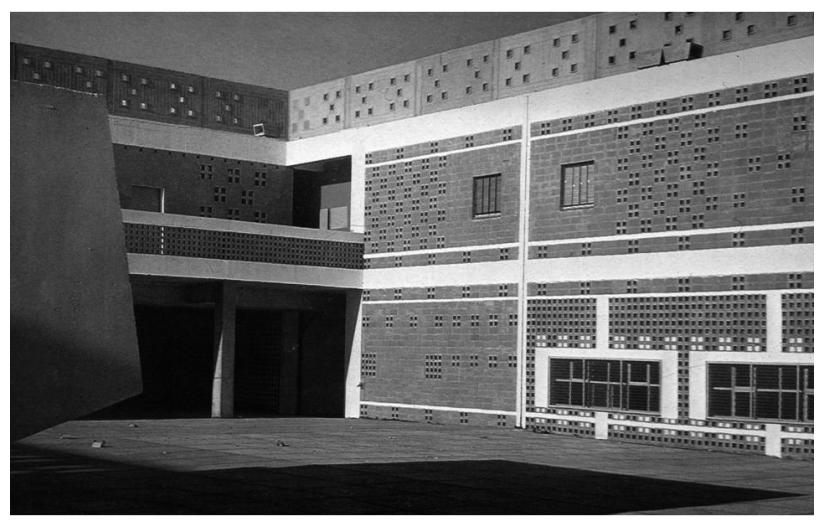

Ill. 1 : Centre Saint Réparatus, architecte Jean Bossu, carte postale antérieure à 1962. (C) Technique et architecture, $\mathrm{n}^{\circ} 329$, février-mars 1980, numéro spécial Algérie.

Entre 1954 et 1958, en seulement quatre ans, l'équipe de Jean de Maisonseul reconstruit presque entièrement une ville détruite à environ $80 \%$, contrairement à la lenteur des reconstructions d'Après-guerre. Orléansville se veut être un exemple.

Au printemps 1955, accompagné de Jean de Maisonseul, Louis Miquel et Roland Simounet, Albert Camus visite Orléansville. Il est frappé par l'ambiance qui règne sur ce vaste chantier. Il note ses impressions et de retour à Paris, il partage son admiration dans un article de L'Express en mai 1955, décrivant le climat chaleureux et humain qui y règne :

"En communauté, sans aucun confort, d'une vie à demi monastique par le dénuement et la sobriété, mais que l'énergie, la lumière, la joie de faire, la camaraderie, remplissent de bonnes jouissances [...]. Loin de nos faux métiers, de nos petits ressentiments, de nos communautés vides ou destructrices, de nos solitudes incomplètes, ils exercent, dans la chaleur du travail créateur, un métier d'homme."

On peut se demander pourquoi Jean de Maisonseul, lui aussi fervent admirateur de Le Corbusier et l'ayant déjà rencontré, n'a pas sollicité le "maître " pour la reconstruction d'Orléansville. Le Corbusier, dont les projets d'urbanisme radical depuis le plan "Voisin » des années 1920 sont souvent refusés (Barcelone, Bogota, La Palice, plan Obus d'Alger), met enfin en ouvre ses idées sur l'urbanisme et l'architecture à Chandigarh. Il y travaille déjà en 1954 au moment du séisme d'Orléansville. Peut-être n'est-il pas disponible à ce moment-là ?

\section{Le début de la guerre d'Algérie et l'apport d'Albert Camus}

Il existe à Alger un cercle d'intellectuels (comédiens, écrivains, journalistes, architectes etc.) proche du parti communiste, ayant lutté aux côtés des républicains espagnols en 1936 puis contre le fascisme durant la Seconde guerre mondiale. À partir de 1954, ils luttent contre les discriminations raciales et militent pour la 
cohabitation des deux peuples nés sur un même territoire. L'art et le théâtre sont leur premier ciment.

Louis Miquel et Roland Simounet se sont engagés aux côtés des nationalistes algériens.

En hiver 1955, pendant le projet du centre culturel de la jeunesse et de sports d'Orléansville, est fondé un comité pour une trêve civile en Algérie dont Louis Miquel sera le co-fondateur avec Albert Camus, dont Roland Simounet et Jean de Maisonseul sont signataires, ce qui vaudra à ce dernier d'être jeté en prison.

La reconstruction d'Orléansville est perçue comme un événement «symbolique ", intervenu au tout début de la guerre, qui permet aux architectes d'essayer de créer un nouvel espoir de cohabitation entre les communautés, l'espoir d'une ville fraternelle et égalitaire chère aux utopies de Camus mais aussi à celles du Mouvement moderne et de Le Corbusier.

\section{Un espoir dans la tourmente : le centre de la jeunesse et de sports Albert-Camus}

Louis Miquel, Roland Simounet: les "enfants du pays"

Né en 1913 à Aïn-Témouchent près d'Oran, Louis Miquel décède en 1986 à Sète en France. Il est à la fois architecte, urbaniste et décorateur de théâtre.

Après la conférence de Le Corbusier en 1931 à Alger, Louis Miquel devient un corbuséen "inconditionnel ", nom que lui attribue le "maître ", après un passage à l'atelier de la rue de Sèvres. À la fin de sa bourse, en 1935, il lui conseillera de partir au Brésil mais Miquel préferera rentrer à Alger dont il se sent plus proche culturellement et où il exercera jusqu'en 1962.

Dès son retour à Alger en 1935, il fonde avec Pierre Miquel, son frère, et Albert Camus, devenu un ami très proche, la troupe de comédiens amateurs du Théâtre du travail, devenu "L'Équipe » en 1938, pour lesquels il réalise des décors avec un grand souci de sobriété et de simplicité (ill. 2). Jean de Maisonseul est à l'initiative du choix de Louis Miquel en tant qu'architecte pour le centre culturel AlbertCamus, comme il l'a été aussi pour l'Aéro-habitat.

Né le 31 août 1927 à Guyotville (aujourd'hui Aïn-Benian près d'Alger), Roland Simounet y passe toute son enfance, puis il étudie et pratique son métier d'architecte jusqu'en 1962 à Alger. Joseph Abram, historien de l'architecture, écrit: "Simounet donne une forme tangible aux aspirations de toute une génération d'architectes, qui voient dans le vernaculaire et la culture populaire un moyen de renouveler la modernité ${ }^{2}$."

Lorsqu'il rejoint Louis Miquel pour la construction du centre Albert-Camus, les notions de "nécessité " et de justesse, qu'il aura apprises lors de son étude sur le bidonville de Mahieddine et qu'il communiquera au CIAM 9 d'Aix-en-Provence en 1953, sont à nouveau une base de travail pour ce projet d'Orléansville après le

2. Gérard Monnier (dir.), Joseph Abram, L'Architecture moderne en France, tome 2. Du chaos à la croissance 1940-1966, Paris, Picard, 1999, p. 276.

Livraisons J'bistoire de l'architecture $n^{\circ} 30$ 


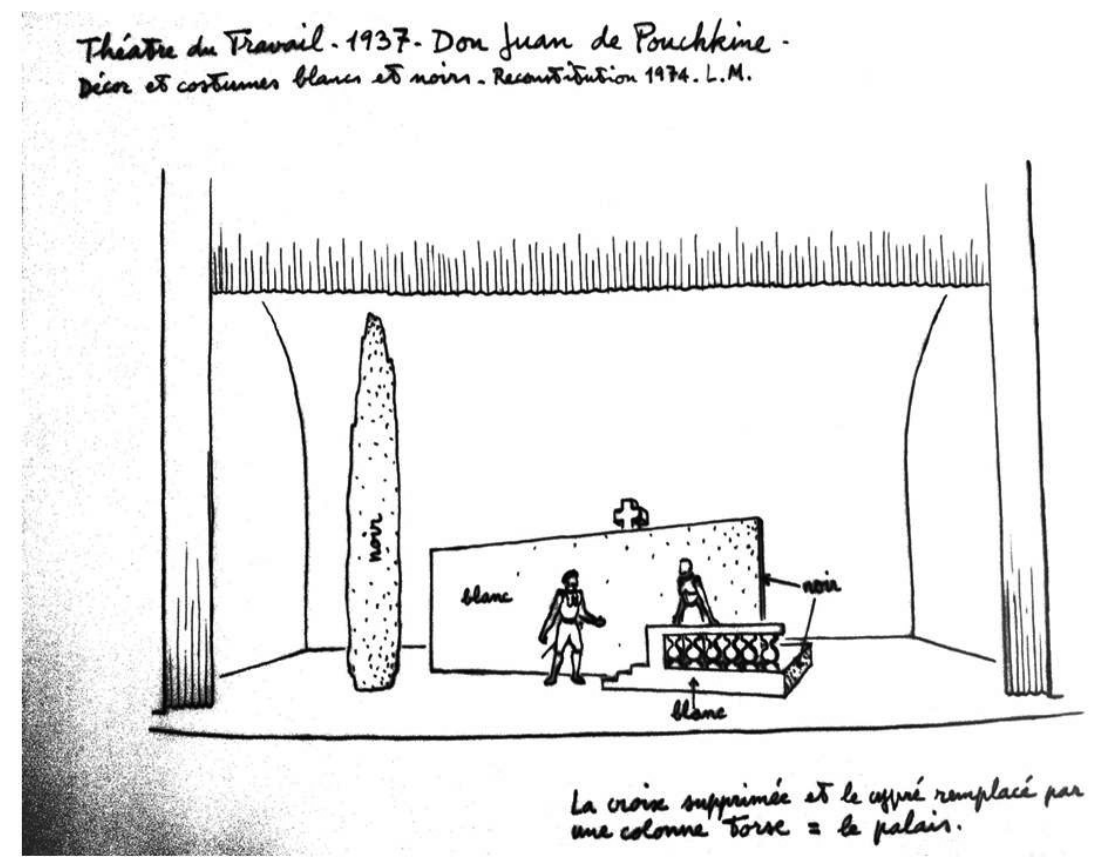

Ill. 2 : Croquis de Louis Miquel pour les décors du théâtre "L’Équipe " et du "Théâtre du travail ", reconstitution de 1974. (C) IFA, fonds Louis Miquel cote Miqlo-B-36 ifa 007 1-9.

séisme de 1954 : un petit projet, mais généreux et juste, tant par la forme que par la taille des bâtiments.

Miquel et Simounet sont proches par leur humanisme, leur éthique professionnelle, l'amour de la terre algérienne et leur admiration pour Le Corbusier, leur "maître" en architecture.

Pour tous les deux, c'est une de leurs dernières réalisations en Algérie. Son élaboration et sa construction couvrent pratiquement toute la durée de la Guerre d'Algérie, commencée une année après le soulèvement de 1954 et inaugurée une année avant l'Indépendance de 1962.

Les qualités humaines des deux hommes vont déteindre sur la qualité architecturale du centre culturel et sportif qu'ils vont édifier ensemble : il s'en dégage une philosophie hédoniste chère à Camus. Le programme du centre culturel prévoyait de rassembler les hommes dans un lieu où la culture l'emporterait sur la division et la haine.

Les conditions du programme: une maison des jeunes et de la culture avant l'heure

Le programme, pensé par de Maisonseul, illustre la combinaison de la culture et du sport, issue des idéaux progressistes d'avant-guerre, mais surtout le principe de l'articulation de plusieurs locaux d'activités culturelles autour d'une salle de théâtre. Le programme que doivent traiter Louis Miquel et Roland Simounet anti- 
cipe le programme des Maisons de la culture qui se développe en France à partir de 1959 avec la création du secrétariat d'État aux Affaires culturelles dirigé par André Malraux. Certains composants du programme qui se développent autour d'une salle de théâtre ainsi que la polyvalence des espaces qui est explicitement demandée, relèvent de ce qui sera le programme phare de la politique d'action culturelle de la France des années soixante.

Au cours d'un séjour d'Albert Camus à Alger au début de l'année 1955, les architectes lui ont demandé de les conseiller sur le choix d'un dispositif scénique. Il leur propose de s'inspirer du théâtre élisabéthain réputé pour son concept très libre par opposition au concept classique soumis à des règles très strictes, qu'il s'agisse de l'architecture du théâtre lui-même, de la mise en scène ou du jeu des comédiens.

La scène élisabéthaine est logée dans un édifice à ciel ouvert, de forme polygonale ou circulaire, formant une arène ; elle est entourée de galeries sur trois étages où se tenait le public. Miquel et Simounet prennent le parti de volumes clairs, simples et épurés, imbriqués les uns dans les autres. Ce décor est réduit à quelques meubles qui posent le lieu et l'action. Le fond de mur devient alors support pour le metteur en scène. L'austérité domine, sans grandiloquence. Louis Miquel écrit à Albert Camus le $1^{\text {er }}$ octobre 1955 pour lui demander son avis sur les dispositifs scéniques de la salle de théâtre du centre.

\begin{abstract}
"La scène pourrait être utilisée aussi dans son dépouillement, en la limitant simplement par des panneaux [...]. Elle pourrait même servir dans sa nudité totale, les acteurs pouvant aller d'un côté à l'autre en passant par les dessous où sont situées les loges d'artistes et des magasins à décors et accessoires. Les scènes latérales, quand elles ne seraient pas utilisées, pourraient servir de promenoirs en y installant des garde-corps amovibles. La salle pourrait être asses rude (matériaux brutes). Les sièges seraient simplement constitués par des planches de bois poli scellés sur les gradins. L'obliquité de ceux-ci me semble nécessaire pour faciliter la visibilité des scènes latérales. »
\end{abstract}

Albert Camus répond le 23 novembre 1955 en lui confirmant qu'il est intéressé par ce projet et la conception scénique de ce théâtre.

\title{
Le chantier
}

Le terrain affecté à l'édification de ce centre est une ancienne pépinière du génie militaire, une vaste cuvette plantée d'arbres magnifiques. Le terrain disponible est en creux, en forme d'amphithéâtre, boisé de pins. Le programme est celui d'un centre d'accueil avec une auberge de jeunesse sur deux étages comprenant des dortoirs pour filles et garçons. Un réfectoire et sa cuisine, le logement du directeur, des salles de travail et de réunion et un laboratoire de photos. Les salles sont conçues avec cette même volonté de simplicité des formes et de clarté des distributions. Ce centre d'accueil est relié au centre culturel par un passage traversant un enclos d'exposition à ciel ouvert, une promenade architecturale qui n'est pas sans rappeler le discours de Le Corbusier et ses rampes (ill. 3). Là, s'ouvre un vaste hall poly- 


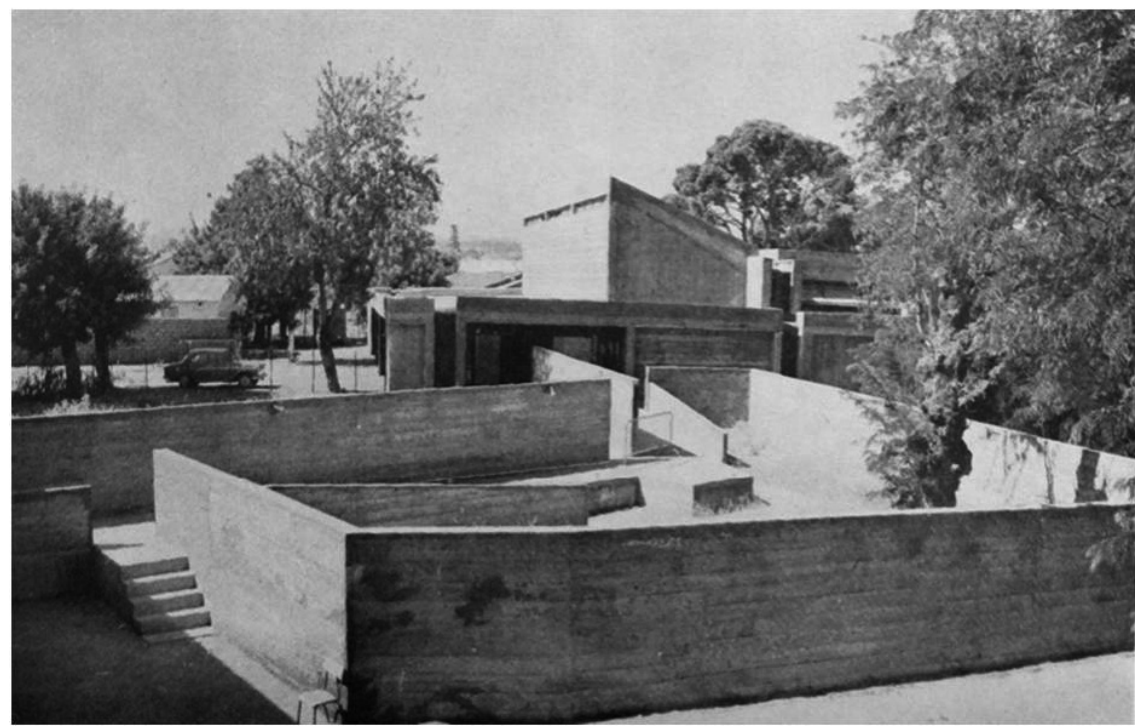

Ill.3 : Enclos d'exposition en plein air reliant le centre d'accueil au centre culturel. (C) IFA, fonds Louis Miquel cote IFA-007 ifa2-10.

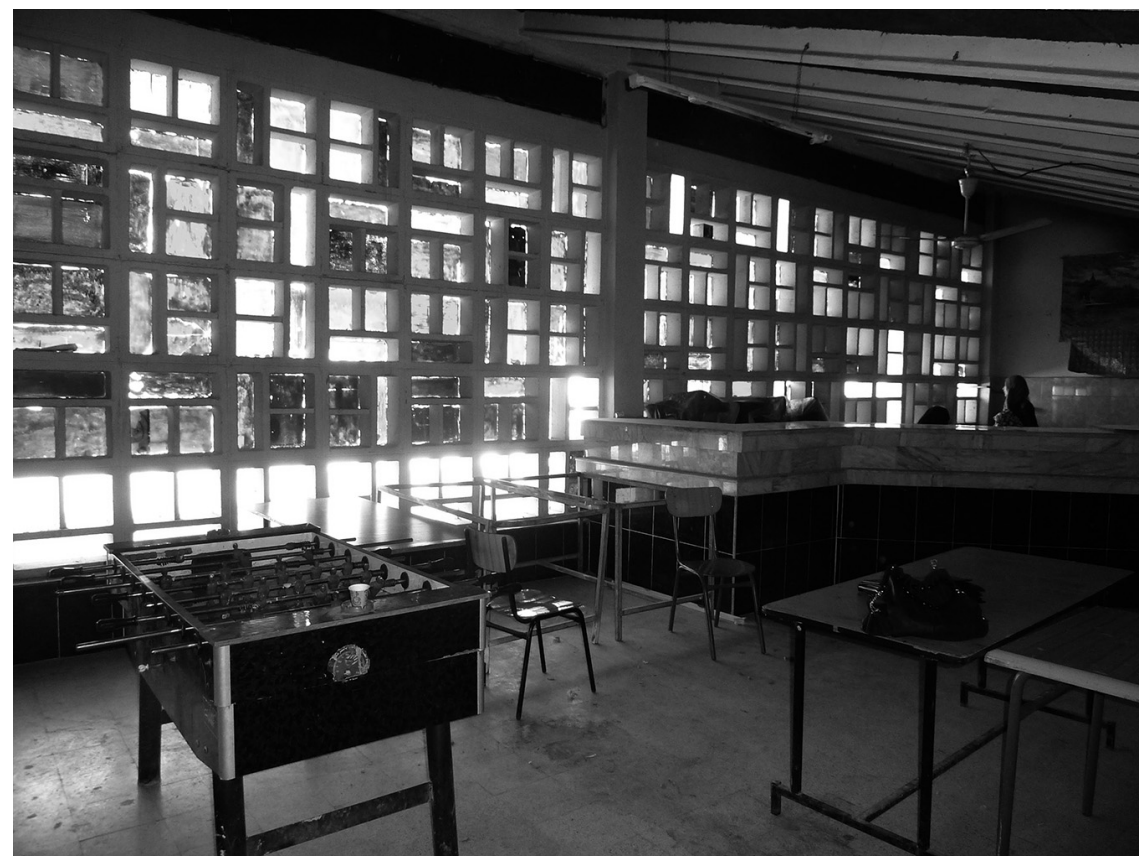

Ill. 4 : Détails des claustras et vitraux. (C) IFA, fonds Louis Miquel cote IFA-007 ifa2-10. 
valent (foyer, salle de jeux, exposition) largement éclairé sur la façade principale par un shed percé de panneaux de claustras donnant l'aspect d'une "cathédrale ", les vitraux étant remplacés par des pans de verres colorés filtrant la lumière (ill. 4). Des impostes au ras du plafond assurent une bonne ventilation. Une petite salle de conférence-bibliothèque sur deux niveaux est reliée au foyer par un grand panneau pivotant ; elle est agrémentée de claustras et de vitraux au sud.

En suivant les conseils de Camus et les principes du théâtre élisabéthain, la scène intérieure est pourvue d'un dispositif scénique fixe formant un seul volume avec la salle : pas de rideau, pas de cache de scène, pas de machinerie, mais uniquement des loggias ou balcons sur divers niveaux en fond de scène et sur un côté de la salle. Des communications multiples entre les loggias, la scène, la salle et les coulisses offrent ainsi une grande diversité de lieux scéniques (ill. 5). L'unité architecturale du volume salle-scène offre une liaison permanente entre le public et les spectateurs. Les gradins de 600 places épousent parfaitement la pente du terrain en vue d'une bonne visibilité. Le décor devenu inutile s'est réduit à quelques éléments situant le lieu et l'action.

Une série de rampes, de passerelles et d'escalier relient ce centre culturel au centre de manifestation en plein air polyvalent (théâtre, concert, chorégraphie, natation) avec des gradins pour 1500 spectateurs. À la manière des théâtres romains en hémicycle et par souci de préserver le plus grand nombre d'arbres, ces gradins utilisent la pente naturelle tout en insérant dans leur construction quelques grands pins qui ne nuisent pas à la visibilité. La piscine réglementaire de 25 mètres de long est incorporée à la scène, elle peut être couverte en partie ou en totalité par des panneaux de bois (ill. 6).

Un terrain de jeux (basket-ball, volley-ball, hand-ball, tennis, piste de course à pied de 400 mètres, sautoirs) est envisagé. Afin de préserver les arbres, la piste de course est déformée. Enfin, des vestiaires collectifs pour les installations sportives sont prévus.

On peut y voir la concrétisation du "théâtre idéal » recherché déjà par Louis Miquel dans ses premières réalisations de décor théâtral avec Camus. Également, dans cette imbrication de plusieurs programmes on pense à Le Corbusier avec son théâtre en plein air sur le toit de l'Unité d'habitations de Firminy, à côté de l'école et du gymnase.

Tous les bâtiments sont en béton banché laissé brut de décoffrage à l'extérieur et à l'intérieur, les claustras apportent un jeu de couleurs primaires contrastant avec la rudesse grise du béton.

\section{La réception du bâtiment}

\section{L'inauguration du centre culturel}

Le 4 avril 1961 est inauguré le centre de la Jeunesse et de sports Albert-Camus à Orléansville. Cette manifestation est avant tout l'occasion de rendre un vibrant hommage à Albert Camus, décédé un an auparavant. Elle rassemble ses amis algériens. 


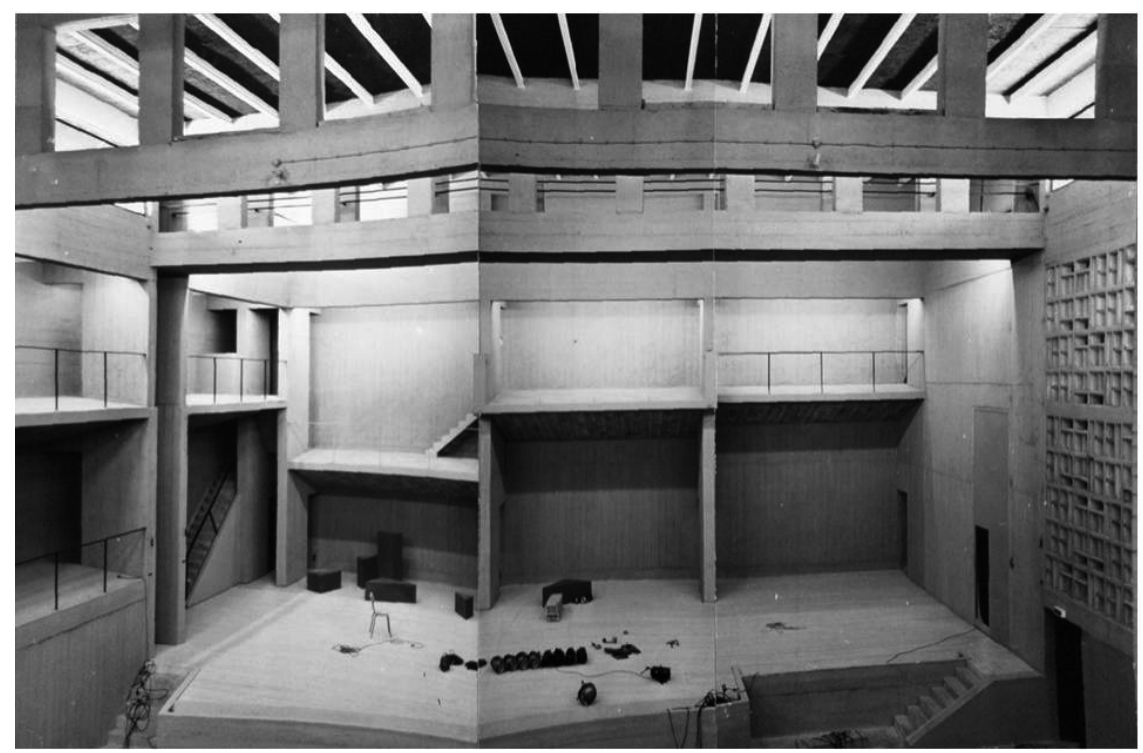

Ill. 5 : Salle de théâtre intérieure, photo de chantier. (C) IFA, fonds Louis Miquel cote IFA-007 ifa2-10.
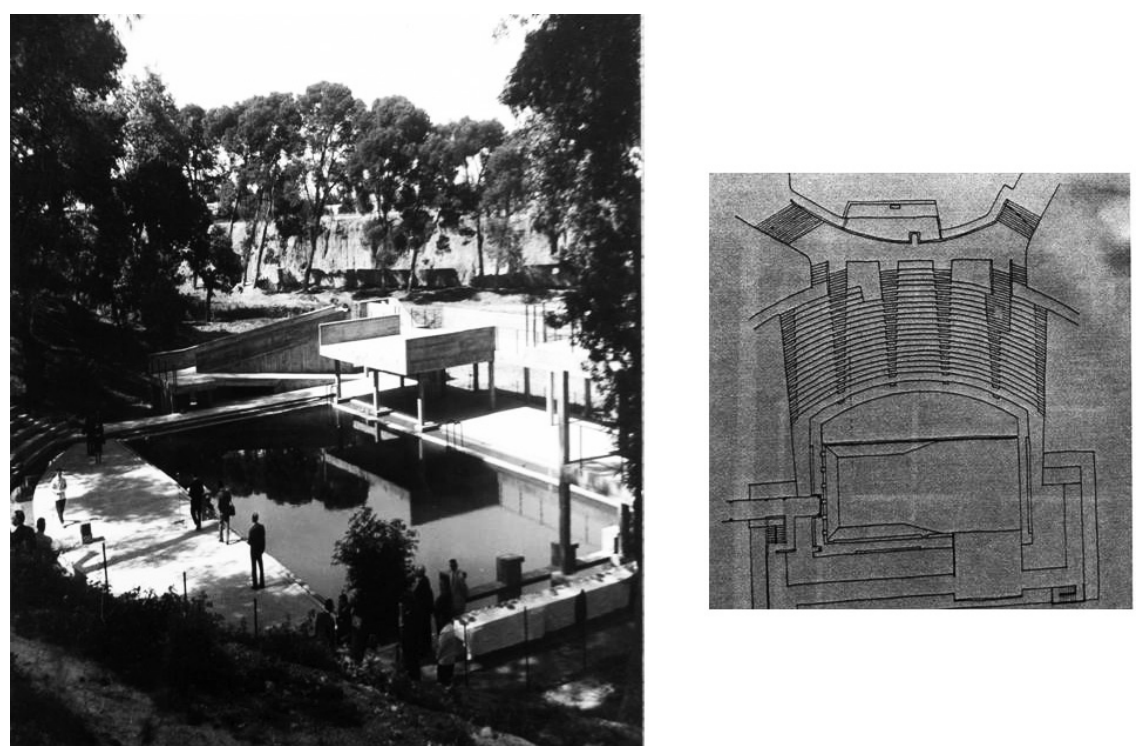

Ill. 6 : Piscine du centre Albert-Camus, 1961 et plan du théâtre de plein air et de la piscine. (C) AMT, fonds Roland Simounet cote 1997017595. 
Après les discours d'inauguration, les participants découvrent l'exposition des peintres et amis de Camus dans la galerie de plein air conçue à cet effet. Ils assistent à la présentation de travaux effectués lors d'un stage d'art dramatique par les instructeurs du " théâtre populaire ", Françoise Becht, Abderrahmane Kaki, Henri Cordreaux et Kamal Babadoun, entre autres. Dans le théâtre en plein air est jouée en arabe La mégère apprivoisée de Shakespeare, mise en scène par Kamal Babadoun (ill. 7).

Quant au contexte et à l'ambiance de cette inauguration, bien qu'agréable et festive, elle a lieu seulement quinze mois avant l'indépendance de l'Algérie. Dans cette atmosphère trouble et chaotique durant laquelle la reconstruction de la ville ne recule pas, les journalistes n'oublient pas de saluer la conception scénique nouvelle pour une activité théâtrale modernisée.

"Une scène dont ont rêvé certainement, ou dont rêvent les metteurs en scène Charles Dullin, Georges Pitoëff, Jacques Copeau ou Vilar ${ }^{3}$."

Le centre culturel a fonctionné un an sous l'administration française, un prospectus de 1961 témoigne des activités théâtrales qui y étaient programmées.

\section{De 1962 à aujourd'hui : un bâtiment en survie}

En 1963, juste après l'indépendance, le nom du centre culturel «AlbertCamus" est remplacé par celui de "Larbi Tebessi ». M. Boudia, directeur du "café littéraire " de Chlef et écrivain, parle "d'une conjoncture historique », il ajoute même que "d'après certains, Cheikh Larbi Tebessi était lié d'amitié avec Albert Camus, car ils avaient peut-être les mêmes vues sur la finalité de la lutte du peuple algérien.»

Des photographies de 1963 attestent que le centre culturel est en pleine activité, la piscine olympique est remplie d'eau et comme préparée pour recevoir une compétition. Le site internet "Culture à Chlef- El-Asnam " tenu par M. Boudia, relate une émission radio retraçant l'historique du centre Larbi Tebessi. En passant en revue les pièces jouées depuis son ouverture en 1961, on note qu'en 1966 la troupe d'El-Asnam remporte le $1^{\text {er }}$ prix du festival d'art dramatique au cinéma "Atlas " à Alger.

Les années "noires" et le lourd tribut payé par les artistes auront des répercussions sur le dynamisme et le bon fonctionnement du centre de la jeunesse et de sports.

Le 30 octobre 1980, la terre tremble à nouveau à El-Asnam. Le séisme, plus violent que celui de 1954, détruit à nouveau la ville à $80 \%$. On compte 2633 morts, des milliers de blessés et de disparus pour une population de 120000 habitants en 1980.

L'architecture nouvelle a souvent mal résisté aux violentes secousses. Au contraire, le centre Albert-Camus, pour lequel Miquel et Simounet avaient prévu des poutres, des chaînages et des voiles antisismiques, ne sera pas détérioré, hormis quelques fissures sans conséquence sur la solidité du bâti. Ce cataclysme a permis une prise

3. L'Écho d'Alger, 5 avril 1961.

Livraisons J'bistoire de l'architecture $n^{\circ} 30$ 


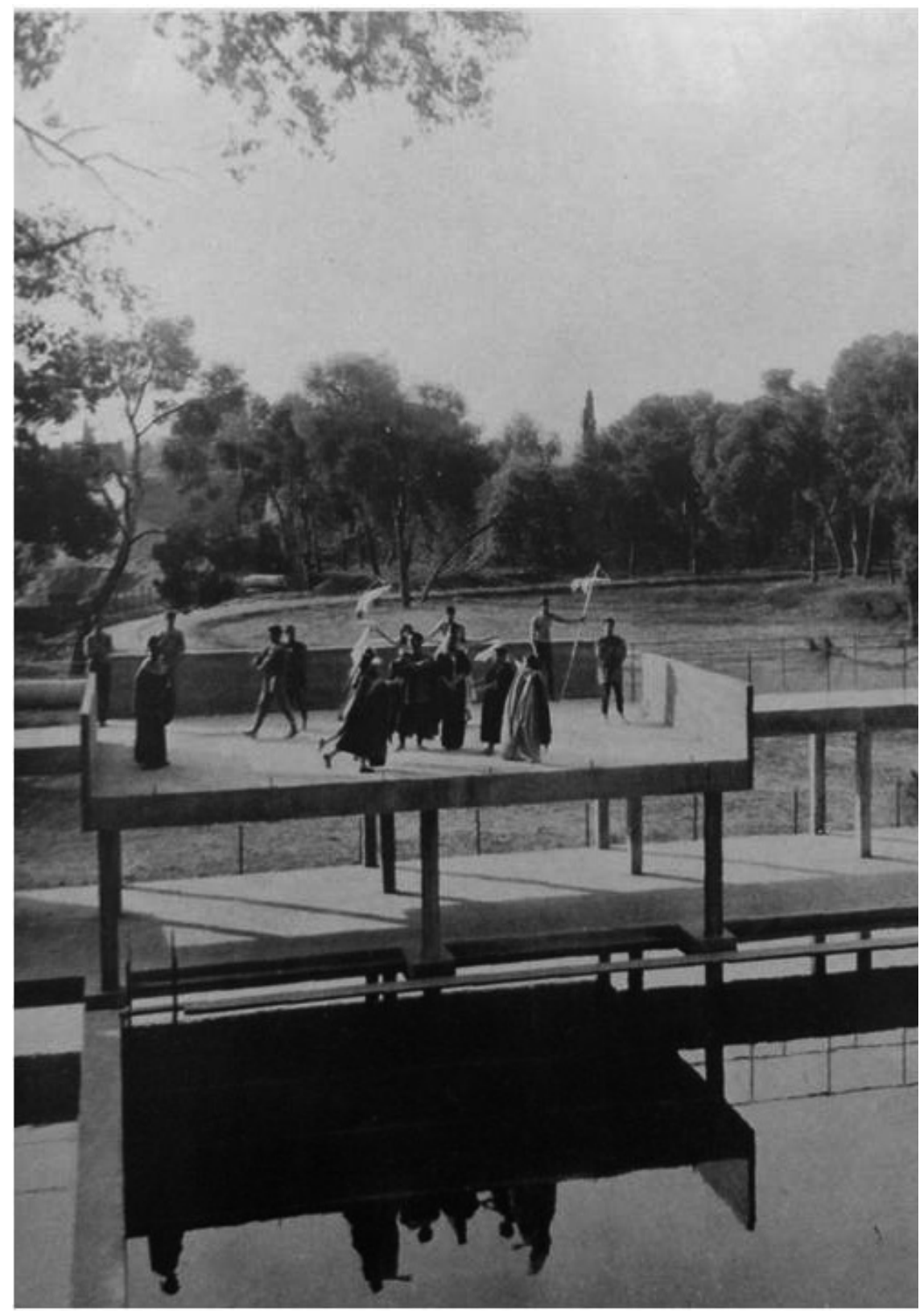

Ill. 7 : Activité théâtrale sur l'une des plates-formes du théâtre en plein air, le 4 avril 1961. (C) IFA, fonds Louis Miquel cote IFA-007 ifa2-10. 
de conscience et l'établissement d'une réglementation parasismique algérienne (le RPA) pour les futures constructions.

Lors d'une visite au centre culturel en avril 2012, nos accompagnateurs nous font savoir qu'après 1980 il n'y a eu aucune modification sur les bâtiments, mais que le bâtiment souffrait d'un manque d'entretien. Ce n'est qu'entre 2002 et 2004 que l'on commence à s'occuper de son état et de sa rénovation. Hormis des modifications à l'intérieur du bâtiment et sur l'entrée principale, la façade principale ne semble pas avoir subi de modifications. On regrette cependant l'occultation des vitraux du théâtre créant autrefois des jeux de lumières, et l'ajout d'un rideau de scène pourtant exclu du projet de Miquel et Simounet car contraire aux principes du théâtre élisabéthain (ill. 8).

En 2012, du centre culturel de la jeunesse et de sports il ne reste que le centre culturel et son théâtre, le théâtre en plein air et sa piscine, et l'aire sportive transformée en terrain de football. Le hall d'exposition en plein air n'est plus en usage, quelques graffitis recouvrent les murs, la piscine n'est plus en fonctionnement, le centre d'accueil est squatté.

Malgré tout, les défenseurs de ce lieu culturel sont bien là, solidaires, et ont réussi à en faire un espace toujours vivant, un refuge pour la jeunesse et un lieu d'espoir et de chaleur humaine avec des moyens très limités mais avec beaucoup d'imagination. On assiste à des répétitions de groupes de musique et à un atelier de confection de marionnettes.

Deux articles, issus du quotidien "El Watan " et datés de novembre 2009 et mai 2012, témoignent de cette "agitation positive " et de son intérêt patrimonial. Le premier présente le centre Larbi Tebessi comme " un site culturel en quête d'un classement national », quant au second il relate la huitième édition du Festival national du théâtre universitaire dont le coup d'envoi est donné au "temple du théâtre dans la région".

En octobre 2014, lors d'une seconde visite, l'architecte Mansour Boucktach, dont le bureau d'études était déjà chargé en 2002 de la rénovation du bâtiment du centre culturel, redémarre une nouvelle campagne de rénovation. Le centre d'accueil, vidé de ses squatters, va redevenir une auberge de jeunesse. L'architecte veut retrouver le souffle et la volonté qui animèrent Louis Miquel et Roland Simounet, tant pour les matériaux, en retrouvant le béton banché originel des façades et en réinstallant les panneaux de claustras colorés, que pour le programme, en restituant l'enclos d'exposition en plein air et en restaurant les terrains de sports. La piscine seule est menacée : certains voudraient la combler, et l'architecte se bat pour lui redonner son utilité et son agrément.

Les espaces extérieurs, pourvus des mêmes eucalyptus de 1961 et d'une végétation associée au béton brut banché des façades du centre culturel, donnent à ce lieu la même magie et sensation d'osmose entre le site et le bâti voulue par ses architectes en 1955. Une architecture "juste ", où l'esprit du lieu est encore présent.

Soraya DU CHAZAUD

historienne de l'architecture 

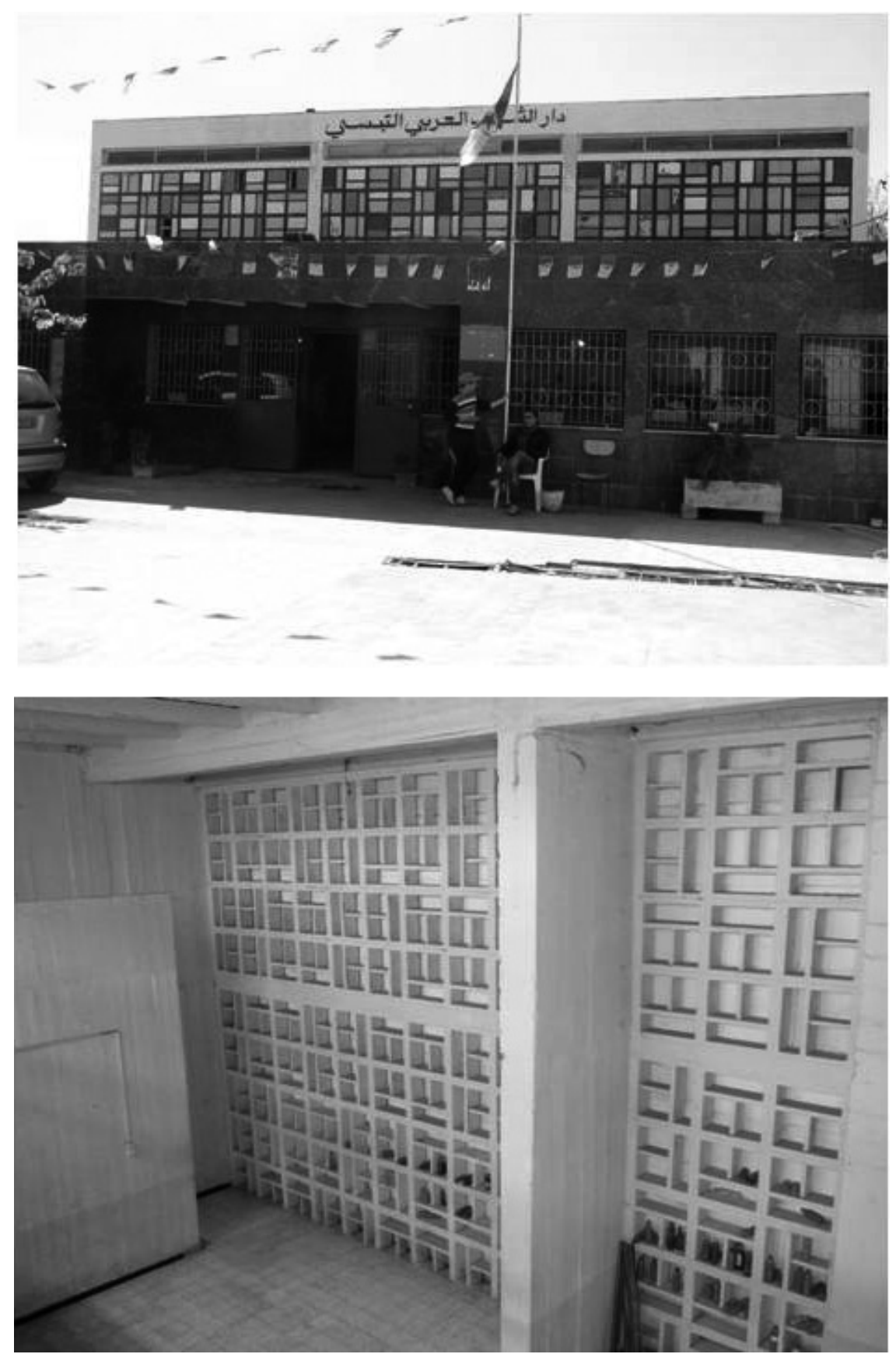

Ill. 8 : Centre culturel Larbi Tebessi, avril 2012. (C) Cliché Soraya du Chazaud. 
